\title{
The relationship between pre-pregnancy BMI, gestational weight gain and neonatal birth weight: a retrospective cohort study
}

\author{
Magdalena Nowak' ${ }^{1}$, Maria Kalwa' ${ }^{1}$, Piotr Oleksy² , Katarzyna Marszalek', \\ Malgorzata Radon-Pokracka ${ }^{1}$, Hubert Huras ${ }^{1}$ \\ ${ }^{1}$ Jagiellonian University Collegium Medicum, Department of Obstetrics and Perinatology, Cracow, Poland \\ ${ }^{2}$ AGH University of Science and Technology, Cracow, Poland
}

\begin{abstract}
Objectives: Maternal pre-pregnancy body mass index (BMI) and gestational weight gain (GWG) have a meaningful impact on pregnancy and perinatal outcomes. The first aim of the study was to analyze the association between pre-pregnancy BMI and the prevalence of small for gestational age (SGA) and large for gestational age (LGA) outcomes. The second aim was to assess the relationship between pre-pregnancy BMI combined with gestational weight gain (GWG) and the prevalence of SGA and LGA measurements. Material and methods: The retrospective cohort study was conducted at Jagiellonian University Hospital in Cracow, Poland from 2016 to 2017. During this time there were 2,123 deliveries. Patients with chronic diseases, multiple pregnancies, fetal defects and incomplete data were excluded. Finally, 474 cases were enrolled. Patients were divided into BMI groups (underweight, normal, overweight and obese) and into GWG groups (inadequate, adequate, excessive). Relationships between maternal BMI, GWG and newborn weight were examined.

Results: There was no statistically significant association between maternal pre-pregnancy BMI and prevalence of SGA measurements. However, underweight women with inadequate GWG showed a higher risk to bear SGA babies (OR 5.2, 95\% $\mathrm{Cl}$ 1.57-17.18). Obese women with adequate GWG had higher risk of bearing LGA newborns (OR 5.48, 95\% $\mathrm{Cl} 1.15-26.13$ ). High BMI correlated with excessive GWG (overweight: OR 3.0, 95\% Cl 1.84-3.87; obese OR 2.45, 95\% Cl 1.1-5.48).

Conclusions: There is a considerable risk of giving birth to a SGA newborn for underweight women with inadequate GWG. There is a statistically significant association between maternal obesity and LGA outcomes. Our study shows that redefining the risks of abnormal neonatal weight considering both pre-pregnancy BMI and gestational weight gain can be useful in providing effective prevention during pregnancy.
\end{abstract}

Key words: BMl; weight gain; SGA; LGA; pregnancy

Ginekologia Polska 2019; 90, 1: 50-54

\section{INTRODUCTION}

Maternal pre-pregnancy body mass index (BMI) and gestational weight gain (GWG) have a meaningful impact on pregnancy and perinatal outcomes. High maternal body weight (HBW) has been shown to increase the risk of stillbirth, diabetes mellitus (DM), hypertension and preeclampsia in pregnancy [1, 2]. Low body weight carries a risk of preterm birth and low neonatal birth weight [3].

The first aim of the study was to analyze the association between pre-pregnancy BMI and the prevalence of small for gestational age (SGA) and large for gestational age (LGA) outcomes. The second aim was to assess the relationship between pre-pregnancy BMI combined with gestational weight gain (GWG) and the prevalence of SGA and LGA measurements. These associations have not been fully analyzed in previous studies. Defining the relationship between maternal body weight and newborn birth weight can be beneficial to attain a better understanding of the underlying causes of abnormal fetal growth.

\section{MATERIAL AND METHODS}

The retrospective cohort study was conducted between November 2016 and November 2017. During this time there were 2.123 deliveries in the Obstetrics and Perinatology 
Department at Jagiellonian University Hospital in Cracow, Poland. In accordance with the inclusion criteria, the study enrolled 561 cases. The data was obtained from electronic medical records without identifiable patient information and kept anonymous. Ethical approval was granted from the Regional Medical Chamber in Cracow. To eliminate other possible causes of abnormal gestational weight, the women who were qualified for the study did not suffer from any chronic diseases; their pregnancy had to be singleton and without complications.

The inclusion criteria were:

1. singleton pregnancy,

2. no maternal chronic diseases,

3. no congenital fetal abnormalities.

\section{Study variables}

BMI was calculated using maternal weight before pregnancy and height. According to WHO criteria, cases were categorized into 4 groups: underweight $\left(<18.5 \mathrm{~kg} / \mathrm{m}^{2}\right)$, normal weight $\left(18.5-24.9 \mathrm{~kg} / \mathrm{m}^{2}\right)$, overweight $\left(25.0-29.9 \mathrm{~kg} / \mathrm{m}^{2}\right)$, obese $\left(>30 \mathrm{~kg} / \mathrm{m}^{2}\right)$ [4]. Gestational weight gain (GWG) was calculated using the mothers' pre-pregnancy weights and the weights on the day of admission to the labor ward. To assess weight gain in relation to pre-pregnancy BMI, American Institute of Medicine Guidelines were used [4]. Total weight gain was divided into the following categories:

- 12.7-18.4 kg for underweight,

- 11.5-16 kg for normal weight,

- 6.8-11.34 kg for overweight,

- 5-9.07 kg for obese.

Therefore, subgroups based on GWG and BMI were made: inadequate GWG, adequate GWG and excess GWG. Newborns' birth weights and gestational age at birth were analyzed according to WHO growth charts [5]. We defined two groups of abnormal growth: SGA (weight below the $10^{\text {th }}$ percentile) and LGA (weight above the $90^{\text {th }}$ percentile).

\section{Statistical analysis}

The data was analyzed using Python 2.7.6 and Statistica version 13.1. Descriptive statistics involved the calculation of mean and standard deviation. ANOVA was used for quan- titative variables with normal distribution and the Kruskall Wallis test for quantitative variables with other than normal distribution. To compare qualitative data, the Chi square test was used. Also, univariate logistic regression was applied. Differences were considered significant when the $p$ value was less than 0.05 .

\section{RESULTS}

The Obstetrics and Perinatology Department at Jagiellonian University Hospital in Cracow, Poland is a major healthcare center that oversaw 561 uncomplicated pregnancies during this period. In the majority of excluded cases, the pregnancies were complicated by gestational diabetes mellitus, hypothyroidism or hypertension. 95 patients were excluded because the weight before delivery was not recorded in the medical documentation. To avoid potential data bias, excluded and included cases were compared. There were no statistically significant demographic or childbirth weight disparities between the groups. Among the 474 women in the study, there were 43 underweight, 313 normal weight, 91 overweight and 27 obese patients.

The basic characteristics of each BMI group: maternal age, gestational weight gain, pregnancy length, parity and neonatal birth weight are presented in Table 1. No meaningful age, parity and pregnancy length differences were observed between the groups. There were statistically significant ( $p<0.05$ ) GWG differences. As the pre-pregnancy BMI increased, the mean GWG decreased. Among the BMI groups, significant differences $(p<0.05)$ were observed for mean neonatal birth weight $(3197 \pm 455 \mathrm{~g}$ for underweight, $3294 \pm 545 \mathrm{~g}$ for normal weight, $3383 \pm 581 \mathrm{~g}$ for overweight and $3368 \pm 760 \mathrm{~g}$ for obese). Although underweight women had the highest mean GWG, the mean neonatal birth weight for that group was the lowest.

Table 2 presents the association between pre-pregnancy $\mathrm{BMI}$ and small for gestational age (SGA) outcomes. There were no statistically significant associations between BMI and SGA frequency.

The analysis presented in Table 3 shows the association between pre-pregnancy BMI and LGA outcomes. Underweight women were $71 \%$ less likely to have a LGA newborn, whereas for obese woman the risk of having a LGA new-

\begin{tabular}{|l|l|l|l|l|l|}
\hline \multicolumn{7}{|l}{ Table 1. Baseline characteristics by body mass index in the trial cohorts } \\
\hline & Underweight & Normal weight & Overweight & Obese & p \\
\hline Maternal age [years] & $30.14 \pm 4.8$ & $31.79 \pm 4.8$ & $32.62 \pm 5.0$ & $31.31 \pm 5.8$ & 0.6 \\
\hline Gestational weight gain [kg] & $14.45 \pm 4.8$ & $13.87 \pm 5.1$ & $11.47 \pm 7.5$ & $8.59 \pm 7.0$ & $<0.001$ \\
\hline Pregnancy length [days] & $272 \pm 10$ & $272 \pm 14$ & $271 \pm 17$ & $268 \pm 20$ & 0.631 \\
\hline Neonatal birth weight [g] & $3197 \pm 455$ & $3294 \pm 545$ & $3383 \pm 581$ & $3368 \pm 760$ & 0.032 \\
\hline Multipara $n$ [\%] & $24(48.98)$ & $188(53.26)$ & $69(63.89)$ & $16(55.17)$ & 0.204
\end{tabular}


Table 2. Odds ratio (OR) and $95 \%$ confidence intervals (Cls) for the associations between pre-pregnancy body mass index (BMI) and small for gestational age (SGA) outcomes

\begin{tabular}{|l|l|l|l|}
\hline BMI category & OR & $\mathbf{9 5 \%} \mathbf{C I}$ & $\mathbf{p}$ \\
\hline Underweight (43) & 2.0 & $0.94-4.25$ & 0.072 \\
\hline Normal weight (313) & 1.0 & - & - \\
\hline Overweight (91) & 0.48 & $0.21-1.11$ & 0.087 \\
\hline Obese (27) & 1.32 & $0.47-3.67$ & 0.595 \\
\hline
\end{tabular}

Table 3. Odds ratio (OR) and $95 \%$ confidence intervals (Cls) for the associations between pre-pregnancy body mass index (BMI) and large for gestational age (LGA) outcomes

\begin{tabular}{|l|l|l|l|}
\hline BMI category & OR & $\mathbf{9 5 \%} \mathbf{C I}$ & $\mathbf{p}$ \\
\hline Underweight (41) & 0.29 & $0.09-0.98$ & 0.045 \\
\hline Normal weight (313) & 1.0 & - & - \\
\hline Overweight (91) & 1.56 & $0.91-2.65$ & 0.103 \\
\hline Obese (27) & 2.67 & $1.18-6.06$ & 0.018 \\
\hline
\end{tabular}

Table 4: Odds ratio (OR) and $95 \%$ confidence intervals (Cls) for the associations between gestational weight gain (GWG) among BMI groups and small for gestational age (SGA) outcomes

\begin{tabular}{|c|c|c|c|c|c|c|c|c|}
\hline \multirow{2}{*}{ BMI category } & \multicolumn{3}{|c|}{ SGA for inadequate weight gain $n=137$} & \multicolumn{3}{|c|}{ SGA for adequate weight gain $n=195$} & \multicolumn{2}{|c|}{ SGA for excessive weight gain $n=142$} \\
\hline & OR & $95 \% \mathrm{Cl}$ & p & OR & $95 \% \mathrm{Cl}$ & p & & $95 \% \mathrm{Cl}$ \\
\hline Underweight & 5.2 & $1.57-17.18$ & 0.006 & 0.61 & $0.13-2.84$ & 0.529 & 2.52 & $0.43-14.64$ \\
\hline Normal weight & 1.0 & - & - & 1.0 & - & - & 1.0 & - \\
\hline Overweight & 0.82 & $0.22-3.17$ & 0.773 & 0.83 & $0.23-3.05$ & 0.779 & 0.17 & $0.02-1.42$ \\
\hline Obese & 3.12 & $0.66-14.68$ & 0.146 & $\begin{array}{l}\text { no } \\
\text { data }\end{array}$ & no data & no data & 1.51 & $0.28-8.14$ \\
\hline
\end{tabular}

Table 5. Odds ratio (OR) and $95 \%$ confidence intervals (Cls) for the associations between gestational weight gain (GWG) among BMl groups and large for gestational age (LGA) outcomes

\begin{tabular}{|c|c|c|c|c|c|c|c|c|}
\hline \multirow{2}{*}{ BMI category } & \multicolumn{3}{|c|}{ LGA for inadequate weight gain } & \multicolumn{3}{|c|}{ LGA for adequate weight gain } & \multicolumn{2}{|c|}{ LGA for excessive weight gain } \\
\hline & OR & $95 \% \mathrm{Cl}$ & p & OR & $95 \% \mathrm{Cl}$ & p & OR & $95 \% \mathrm{Cl}$ \\
\hline Underweight & 0.37 & $0.04-3.09$ & 0.355 & 0.43 & $0.1-1.98$ & 0.287 & no data & no data \\
\hline Normal weight & 1.0 & - & - & 1.0 & - & - & 1.0 & - \\
\hline Overweight & 1.42 & $0.45-4.44$ & 0.548 & 2.05 & $0.79-5.3$ & 0.135 & 1.32 & $0.58-3.01$ \\
\hline Obese & 2.89 & $0.62-13.51$ & 0.174 & 5.48 & $1.15-26.1$ & 0.032 & 1.63 & $0.44-6.03$ \\
\hline
\end{tabular}

Table 6.0dds ratio (OR) and 95\% confidence intervals (Cls) for the associations between pre-pregnancy body mass index (BMI) and gestational weight gain (GWG)

\begin{tabular}{|l|l|l|l}
\hline Inadequate GWG & OR & $\mathbf{9 5 \%} \mathbf{C l}$ & $\mathbf{p}$ \\
\hline Underweight & 0.88 & $0.44-1.74$ & 0.704 \\
\hline Normal weight & 1.0 & - & - \\
\hline Overweight & 1.33 & $0.77-2.28$ & 0.307 \\
\hline Obese & 1.0 & $0.3-3.3$ & 0.995 \\
\hline Excessive GWG & $\mathbf{O R}$ & $\mathbf{9 5 \%} \mathbf{C l}$ & $\mathbf{p}$ \\
\hline Underweight & 0.7 & $0.31-1.58$ & 0.389 \\
\hline Normal weight & 1.0 & - & - \\
\hline Overweight & 3.0 & $1.84-4.87$ & $<0.001$ \\
\hline Obese & 2.45 & $1.1-5.48$ & 0.028 \\
\hline
\end{tabular}

born was 2.67 greater than the risk for women with normal pre-pregnancy BMI.

The analysis of an association between gestational weight gain (GWG) and SGA outcomes is presented in Table 4. The difference between the consequences of inadequate or excessive weight gain for women with different BMI determined the need to analyze each GWG-BMI subgroup separately. There was a statistically significant risk for underweight women with inadequate weight gain to have a newborn classified as SGA (OR 5.2, 95\% Cl 1.57-17.18).

The results presented in Table 5 show that there was a relationship between LGA newborns and adequate GWG. We observed a statistically significant higher risk of a LGA newborn for obese women (OR 5.48, 95\% Cl 1.15-26.1).

To define the tendencies of abnormal weight gain during pregnancy we compared GWG in the normal BMI group with other BMI groups. The results are shown in Table 6. There was no specific association between inadequate GWG and pre-pregnancy BMI. We observed that high BMI correlated with excessive GWG (for overweight: OR 3.0, 95\% CI 1.84-4.87, for obese OR $2.45,95 \% \mathrm{Cl} 1.1-5.48$ ).

\section{DISCUSSION}

Abnormal pre-pregnancy BMI is a serious healthcare problem in Europe. The Euro Perinatal Health Report has shown that the average European prevalence of overweight 
and obese women is about 30-37\% [6]. In Poland, the proportion is close to $25 \%$ which is the lowest result among European countries. However, this is still a quarter of all Polish pregnant women. According to the Euro Perinatal Health Report, a low pre-pregnancy BMI prevalence of about 9\% in Poland is the highest amount in the study.

Birth weight is an important factor for neonatal outcomes. Both too low and too high weights may cause serious complications. There are numerous factors contributing to intrauterine growth such as genetic predisposition, congenital fetal abnormalities, maternal diseases, lifestyle, addictions and drugs taken during pregnancy. The lifestyle affecting maternal weight and gestational weight gain is a modifiable risk factor and for that reason we studied its relation to neonatal birth weight.

Small for gestational age (SGA) is defined as a weight below the $10^{\text {th }}$ percentile for gestational age. The prevalence of SGA measurements in Europe ranges from 5\% to 15\% [7]. SGA outcomes are associated with severe complications such as decreased oxygen level, hypoglycemia, hypothermia, polycythemia and developmental delay $[6,8]$.

Large for gestational weight (LGA) is defined as a weight above the $90^{\text {th }}$ percentile for gestational age. The overall prevalence for LGA measurements in Europe is 5-20\% [9]. Newborns defined as LGA are prone to adverse obstetric outcomes such as shoulder dystocia, chorioamnionitis, postpartum hemorrhage and longer hospital stay [10].

According to studies concerning the relationship between SGA measurements and BMI, there was a positive association between having a SGA newborn and the mother being underweight [11-13]. However these studies did not concern gestational weight gain and none have divided BMI groups into BMI-GWG subgroups. Without such analysis, the risk that the observed women did not fulfill the criteria of adequate weight gain cannot be ruled out.

In our study, the results did not show an association between pre-pregnancy underweight women and an increased risk of SGA newborns. Although low BMI itself was not determined as a risk factor, there was a considerable risk of SGA outcomes for underweight women who have not reached the recommended level of GWG. The mean GWG among underweight woman was $14.45 \mathrm{~kg}$ which is a result almost $2 \mathrm{~kg}$ higher than the lower recommended GWG range. These findings allow us to propose a hypothesis that among SGA risk factors, being underweight should be considered simultaneously with inadequate weight gain.

Furthermore, we observed a growing association between BMI and LGA outcomes which was also observed in other studies $[14,15]$. Underweight women were less likely to have a LGA newborn while obese mothers had a higher risk of a LGA newborn. Being overweight also was a risk factor for a LGA outcome, but the result was statisti- cally insignificant. Those tendencies were observed also among women divided into GWG subgroups, but the only statistically significant risk of an LGA outcome was seen for obese women with adequate GWG. These findings suggest that the risk of a LGA newborn for obese women can be decreased by reducing the recommended weight gain. Without gestational weight gain recommendations made for the Polish population, it is difficult to verify the statement. Therefore, such a result should initiate further investigations in the Polish population, especially since we have found no Polish studies with which to compare the GWG results and assess the bias in our represented groups. A lower risk of LGA outcomes among obese women with excessive GWG was an unexpected finding. It could be explained as a consequence of increasing obesity which affects fetal growth, but this question should be investigated in separate research.

The analysis of GWG among BMI groups provides our findings with weight gaining tendencies. Underweight women were even more likely to achieve recommended GWG than women with normal weight. Such associations may help explain missing relationships between underweight women and SGA outcomes in our research. The predisposition of overweight and obese women to exceed the recommended GWG should be noted as a serious healthcare problem. According to previous studies, there was no definitive statement about which interventions for excessive weight gain during pregnancy can diminish neonatal and maternal complications [16]. As the tendency to be overweight or obese increases among pregnant women, the problem should be further investigated.

A main limitation of the study was the difficulty in finding women who fitted the strict criteria, especially in the groups with abnormal BMI. However, a major strength of our study was the alternative method of data analysis based on BMI groups and BMI-GWG subgroups. Such a selection strategy diminished the potential risk of data bias and provided us with broader insight into the relations between abnormal neonatal birth weight and maternal risk factors.

\section{CONCLUSIONS}

No statistically significant association between maternal pre-pregnancy BMI and prevalence of SGA newborns was found. There was a clear association between maternal obesity and LGA outcomes, especially when obesity was connected with adequate gestational weight gain. This observation indicates a need to verify if the American recommendations fit the Polish population. There was a considerable risk of SGA for underweight women who have not reached the recommended level of GWG. In conclusion, our study showed that redefining the risks of abnormal neonatal weight by considering both pre-pregnancy BMI and GWG may be useful in providing effective prevention during pregnancy. 


\section{REFERENCES:}

1. Magann EF, Doherty DA, Sandlin AT, et al. Pre-pregnancy body mass index and pregnancy outcomes. Int J Gynaecol Obstet. 2006; 95(3): 242-247, doi: 10.1016/j.ijgo.2006.06.021, indexed in Pubmed: 17007857.

2. Kazemian E, Sotoudeh G, Dorosty-Motlagh AR, et al. Maternal obesity and energy intake as risk factors of pregnancy-induced hypertension among Iranian women. J Health Popul Nutr. 2014; 32(3): 486-493, indexed in Pubmed: 25395911.

3. Han Z, Mulla S, Beyene J, et al. Knowledge Synthesis Group. Maternal underweight and the risk of preterm birth and low birth weight: a systematic review and meta-analyses. Int J Epidemiol. 2011; 40(1): 65-101, doi: 10.1093/ije/dyq195, indexed in Pubmed: 21097954.

4. The American College of Obstetricians and Gynecologists Commitee Opinion Number 548. January 2013 (Reaffirmed 2016)(548).

5. Kiserud T, Piaggio G, Carroli G, et al. The World Health Organization Fetal Growth Charts: A Multinational Longitudinal Study of Ultrasound Biometric Measurements and Estimated Fetal Weight. PLoS Med. 2017; 14(1): e1002220, doi: 10.1371/journal.pmed.1002220, indexed in Pubmed: 28118360.

6. Tenovuo A. Neonatal complications in small-for-gestational age neonates. J Perinat Med. 1988; 16(3): 197-203, indexed in Pubmed: 3264853.

7. Ruiz M, Goldblatt P, Morrison J, et al. Mother's education and the risk of preterm and small for gestational age birth: a DRIVERS meta-analysis of 12 European cohorts. J Epidemiol Community Health. 2015; 69(9): 826-833, doi: 10.1136/jech-2014-205387, indexed in Pubmed: 25911693.

8. Doctor BA, O'Riordan MA, Kirchner $\mathrm{HL}$, et al. Perinatal correlates and neonatal outcomes of small for gestational age infants born at term gestation. Am J Obstet Gynecol. 2001; 185(3): 652-659, doi: 10.1067/mob.2001.116749, indexed in Pubmed: 11568794.

9. Stotland $\mathrm{NE}$, Caughey $\mathrm{AB}$, Breed $\mathrm{EM}$, et al. Risk factors and obstetric complications associated with macrosomia. Int J Gynaecol Obstet. 2004;
87(3): 220-226, doi: 10.1016/j.ijgo.2004.08.010, indexed in Pubmed: 15548393.

10. Chiavaroli V, Castorani V, Guidone $P$, et al. Incidence of infants born small- and large-for-gestational-age in an Italian cohort over a 20-year period and associated risk factors. Ital J Pediatr. 2016; 42: 42, doi: 10.1186/s13052-016-0254-7, indexed in Pubmed: 27117061.

11. Ricci E, Parazzini F, Chiaffarino F, et al. Pre-pregnancy body mass index maternal weight gain during pregnancy and risk of small-for-gestational age birth: results from a case-control study in Italy. J Matern Fetal Neonatal Med. 2010; 23(6): 501-505, doi: 10.3109/14767050903216025, indexed in Pubmed: 19724969.

12. Fujiwara K, Aoki S, Kurasawa K, et al. Associations of maternal pre-pregnancy underweight with small-for-gestational-age and spontaneous preterm birth, and optimal gestational weight gain in Japanese women. J Obstet Gynaecol Res. 2014; 40(4): 988-994, doi: 10.1111/jog.12283, indexed in Pubmed: 24428432.

13. Yu Z, Han S, Zhu J, et al. Pre-pregnancy body mass index in relation to infant birth weight and offspring overweight/obesity: a systematic review and meta-analysis. PLoS One. 2013; 8(4): e61627, doi: 10.1371/journal. pone.0061627, indexed in Pubmed: 23613888.

14. Ehrenberg HM, Mercer BM, Catalano PM. The influence of obesity and diabetes on the prevalence of macrosomia. Am J Obstet Gynecol. 2004; 191(3): 964-968, doi: 10.1016/j.ajog.2004.05.052, indexed in Pubmed: 15467573.

15. Verma A, Shrimali L. Maternal body mass index and pregnancy outcome. J Clin Diagn Res. 2012; 6(9): 1531-1533, doi: 10.7860/JCDR/2012/4508.2551, indexed in Pubmed: 23285448.

16. Muktabhant $B$, Lumbiganon $P$, Ngamjarus $C$, et al. Interventions for preventing excessive weight gain during pregnancy. Cochrane Database Syst Rev. 2012(4): CD007145, doi: 10.1002/14651858.CD007145.pub2, indexed in Pubmed: 22513947. 